\title{
Primary Care Referral Perspectives in Pediatric Failure to Thrive and Outcomes From a Diagnostic Clinic
}

\author{
Grace L. Lisius ${ }^{\mathrm{a}, \mathrm{c}}$, Tony R. Tarchichi ${ }^{\mathrm{b}}$
}

\begin{abstract}
Background: Decades of data demonstrate the overwhelming rates of failure to thrive (FTT) nutritional causes (80-90\%) even in patients with complex medical conditions and diagnoses. Primary care providers (PCPs), the initial FTT evaluators, have been underrepresented in the FTT literature, despite their pivotal role in overseeing management. Our purpose was to assess PCPs' FTT referral strategies and their concerns for their FTT patients referred to an academic pediatrician diagnostic clinic.
\end{abstract}

Methods: We surveyed PCPs who referred FTT patients to a diagnostic clinic (the Diagnostic Referral Group (DRG)) for their main concern prompting referral, and next management if consult was unavailable. Chart review determined the hospitalization rates of FTT patients, and DRG interventions.

Results: Of the $81 \mathrm{PCP}$ responses, $66.7 \%$ were most concerned with an uncertain underlying diagnosis, contrasting with the $20.6 \%$ of patients that DRG providers suspected to have an organic etiology. PCPs' next preferable management was subspecialist referral $(58.0 \%)$ and hospitalization $(22.2 \%)$. DRG providers most commonly recommended nutritional interventions such as increasing caloric density, decreased snacking and juice, and structured meals for $72.4 \%$ of patients.

Conclusions: This study suggests that FTT due to nutritional causes continues to be a challenging diagnosis for PCPs. More investigation is needed to identify interventions to empower primary providers to diagnose nutritional causes, such as practical standardized assessments to evaluate the nutritional and psychosocial FTT contributors.

Keywords: Failure to thrive; Primary care; Nutrition

Manuscript submitted October 24, 2020, accepted November 18, 2020

Published online February 5, 2021

anternal Medicine Residency, University of Pittsburgh Medical Center, 200 Lothrop St \# N713, Pittsburgh, PA 15213, USA

bDepartment of Pediatrics, Children's Hospital of Pittsburgh, University of Pittsburgh Medical Center, Faculty Pavilion-Lawrenceville, Room 3127, 4401 Penn Avenue, Pittsburgh, PA 15224-1334, USA

${ }^{\mathrm{c} C o r r e s p o n d i n g ~ A u t h o r: ~ G r a c e ~ L . ~ L i s i u s, ~ I n t e r n a l ~ M e d i c i n e ~ R e s i d e n c y, ~ U n i-~}$ versity of Pittsburgh Medical Center, 200 Lothrop St \# N713, Pittsburgh, PA 15213, USA. Email: lisiusgl@upmc.edu

doi: https://doi.org/10.14740/ijcp419

\section{Introduction}

Despite overwhelming rates, $80-90 \%$, of nutritional pediatric failure to thrive (FTT), nutrition evaluation is not routine prior to extensive testing and subspecialty referral [1-4]. FTT management is challenged by the absence of consensus diagnostic criteria and lack of a practical nutritional assessment tool in clinics $[3,4]$. The importance of nutritional causes and low utility of exhaustive testing have been shown in both populations with underlying medical conditions and otherwise healthy children [4-7]. Primary care providers (PCPs), the initial evaluators of FTT patients, have not been included in the FTT management research conversation. Understanding PCP's concerns will enable development of targeted tools and interventions to identify and manage children with FTT, streamlining the $5-10 \%$ of outpatient pediatric visits for poor weight gain [2]. Such efficiency changes have sizeable implications on primary care workflow, and on the patients as FTT has lasting consequences on physical and intellectual development.

This study investigated: 1) The concerns and management strategies of PCPs who referred to a diagnostic clinic, the Diagnostic Referral Group (DRG) at Children's Hospital of Pittsburgh (CHP); and 2) The hospitalization rates and interventions for FTT patients at a diagnostic consult. At the DRG, academic pediatricians provided outpatient care to patients with complex multisystem diseases [8,9]. One third of referrals are for poor weight gain.

\section{Materials and Methods}

\section{Community physician survey}

We surveyed community primary care physicians (Supplementary Material 1, www. theijcp.org ) who referred $<18$ years old for DRG evaluation of FTT evaluated from January 1, 2012 to December 31, 2016, identified by the International Classification of Diseases (ICD)-9 codes $783.0-783.43$ and ICD-10 codes R62.51, R63.5, and R63.8. Respondents were excluded if they had referred a patient whose record was not included in the study, were a subspecialist practitioner, or submitted an anonymous survey. Fax and email survey investigated next patient management if DRG appointments were unavailable, using a 4-point qualitative likelihood scale of descending preference: "very likely", "moderately likely", "not at all likely", and "not selected", among the options: hospi- 
Table 1. Characteristics of All Patients With FTT, Evaluated by the DRG for FTT Between January 1, 2012 and December $31,2016$.

\begin{tabular}{|c|c|c|c|c|}
\hline & All FTT patients & $\begin{array}{l}\text { Patients with known } \\
\text { genetic or congeni- } \\
\text { tal diagnosis }\end{array}$ & $\begin{array}{l}\text { Patients with sus- } \\
\text { pected genetic or } \\
\text { congenital diagnosis }\end{array}$ & $\begin{array}{l}\text { Patients with no } \\
\text { suspected genetic or } \\
\text { congenital diagnosis }\end{array}$ \\
\hline Number of patients & 257 & $41(15.9 \%)$ & $53(20.6 \%)$ & $163(63.4 \%)$ \\
\hline Average age & 30.7 months ( 2.5 years) & 57.9 months (4.8 years) & 27.4 months (2.3 years) & 24.9 months (2.1 years) \\
\hline Standard deviation & 39.79 months & 56.7 months & 34.89 months & 33.05 months \\
\hline Median age & 17 & 36 & 16 & 15 \\
\hline$\%$ Male & 59.9 & 63.4 & 69.8 & 55.8 \\
\hline Average weight-for-age z-score & 3.01 & 3.68 & 3.18 & 2.79 \\
\hline Standard deviation & 1.18 & 1.84 & 1.02 & 0.94 \\
\hline Average number of DRG visits & 1.48 & 2.24 & 1.83 & 1.17 \\
\hline
\end{tabular}

The median and quartiles are included for age due to a large positive skew to these data. Percentages given are with respect to total FTT patients or with respect to the subgroup considered. FTT: failure to thrive; DRG: Diagnostic Referral Group.

talization for FTT, a subspecialty consult, and only continuing outpatient care from their office. "Very likely" represented the next preferred management. Physicians then selected the most important patient and logistical factors for their decision to consult the DRG. If a physician had referred multiple patients, they were asked to respond with a response averaging the scenarios. Survey responses were not directly compared to referred patient outcomes. This survey was conducted in compliance with the ethical standards of the responsible institution on human subjects.

\section{FTT patient chart review}

On retrospective chart review, referred patients were included in our review for FTT of undetermined etiology if they met FTT criteria of weight for age $<$ third percentile, or crossing 2 centiles down on their growth curve, and excluded from the study if they did not meet these weight-for-age criteria, had a DRG visit not addressing FTT, or had a hospitalization for FTT within 1 year prior to the visit. Our primary outcome was hospitalization primarily for FTT within 6 months of a DRG visit. Secondary outcomes included DRG interventions, laboratory tests, subspecialty referrals and change in z-score weight-for-age [10]. We accommodated the heterogeneous FTT patient population by performing subgroup analyses, dividing patients on the basis of DRG provider impressions in documentation: no suspected underlying genetic or congenital abnormality to explain the FTT, a suspected but undiagnosed genetic or congenital abnormality, and a previously diagnosed genetic or congenital abnormality (Table 1). Institutional review board approval for the retrospective chart review was obtained through the University of Pittsburgh prior to initiation of data collection. We used a $\chi^{2}$ test for independence on categorical data, three-way tests performed among the subgroups and two-way comparing one category to the average of the other two. These two methods were used to test for whether the three samples were significantly distinct or if there was a single outlying sample. We compared our quantitative data such as age or average number of associated medical conditions using a two sample $t$-test.

\section{Results}

Survey response rate was $84.4 \%$. Of the 108 responses, 81 were included as PCPs (Table 2). Most preferred referral to a subspecialist (58\%, Fig. 1a). If DRG diagnostic consults were unavailable, $22.2 \%$ would have hospitalized their patients (Fig. 1a). Least preferred was continuing only outpatient care (13.6\%). Most PCPs (66.7\%) were concerned about an uncertain underlying diagnosis, with FTT severity (37.0\%) and complex multisystem disease $(33.3 \%)$ of subsequent priority (Fig. 1b).

Records for 257 of 426 patients were included (Table 1), with $193(75.0 \%)$ seen for a single consultation visit without DRG longitudinal follow-up. The majority of patients were as-

Table 2. Characteristics of Included Physicians (Community Physicians Surveyed) Who Referred Patients With FTT to the DRG for FTT Evaluation.

\begin{tabular}{llll}
\hline Specialties & Respondents & Degree & Respondents \\
\hline Pediatrics & $58(72 \%)$ & MD & $73(90 \%)$ \\
Family medicine & $21(26 \%)$ & DO & $8(10 \%)$ \\
Medicine-pediatrics & $2(2 \%)$ & & \\
\hline
\end{tabular}

Percentages given are respective to all included physician responses. MD: doctor of medicine; DO: doctor of osteopathic medicine; FTT: failure to thrive; DRG: Diagnostic Referral Group. 
a

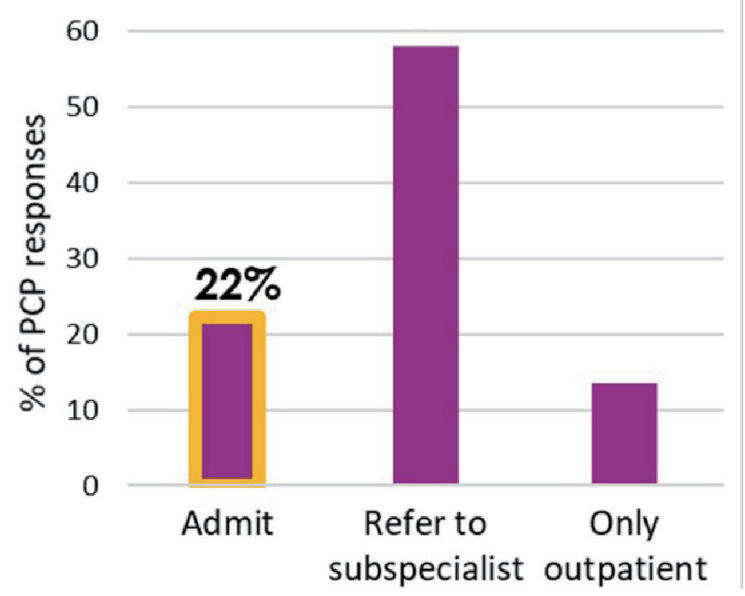

b

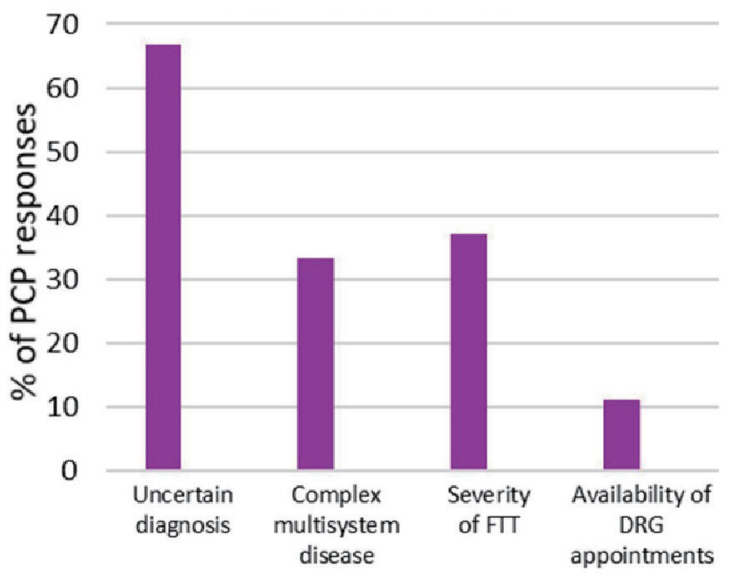

Figure 1. (a) PCPs' most likely next management. (b) Most important factor for DRG referral. PCP: primary care provider; FTT: failure to thrive; DRG: Diagnostic Referral Group.

sessed by the DRG providers as not likely to have a significant underlying medical diagnosis, genetic or congenital abnormality contributing to their FTT. Patients with known significant past medical history carried heterogeneous diagnoses, most commonly congenital heart disease (20 patients), cleft palate (15 patients), associated genetic syndromes (11 patients), and cerebral palsy (six patients) presented more severely underweight $(\mathrm{z}=-3.68, \mathrm{P}=0.028)$, and in which the $\mathrm{DRG}$ suspected an underlying diagnosis presented less underweight $(\mathrm{z}=-2.79$, $\mathrm{P}=0.035)$. Of all patients, $14(5.4 \%)$ were hospitalized for FTT within 6 months of a visit. Patients who were hospitalized were initially more underweight $(\mathrm{z}=-3.74,-2.97, \mathrm{P}=0.030)$. Only patients suspected to have underlying undiagnosed disease showed worsening of weight-for-age z-score prior to ad- mission.

Most patients had improved weight-for-age z-score, 72 $(76.6 \%)$ of the 94 patients with multiple visits or weights. Interventions were most commonly utilized, in 186 (72.4\%), higher in patients without suspected underlying conditions (Figs. 2, 3). Recommendations were nutritional and behavioral: increasing caloric intake and caloric density, keeping a calorie diary, utilizing structured mealtimes, and decreasing snacking and juice (Fig. 3a).

Laboratory investigation was pursued in $136(52.9 \%)$, with fewer tests in patients with known conditions (Fig. 2). Most common laboratory testing included complete blood count (CBC) (57.3\%), electrolyte panel (56.6\%), thyroid panel (27.6\%), and celiac panel (19.5\%).

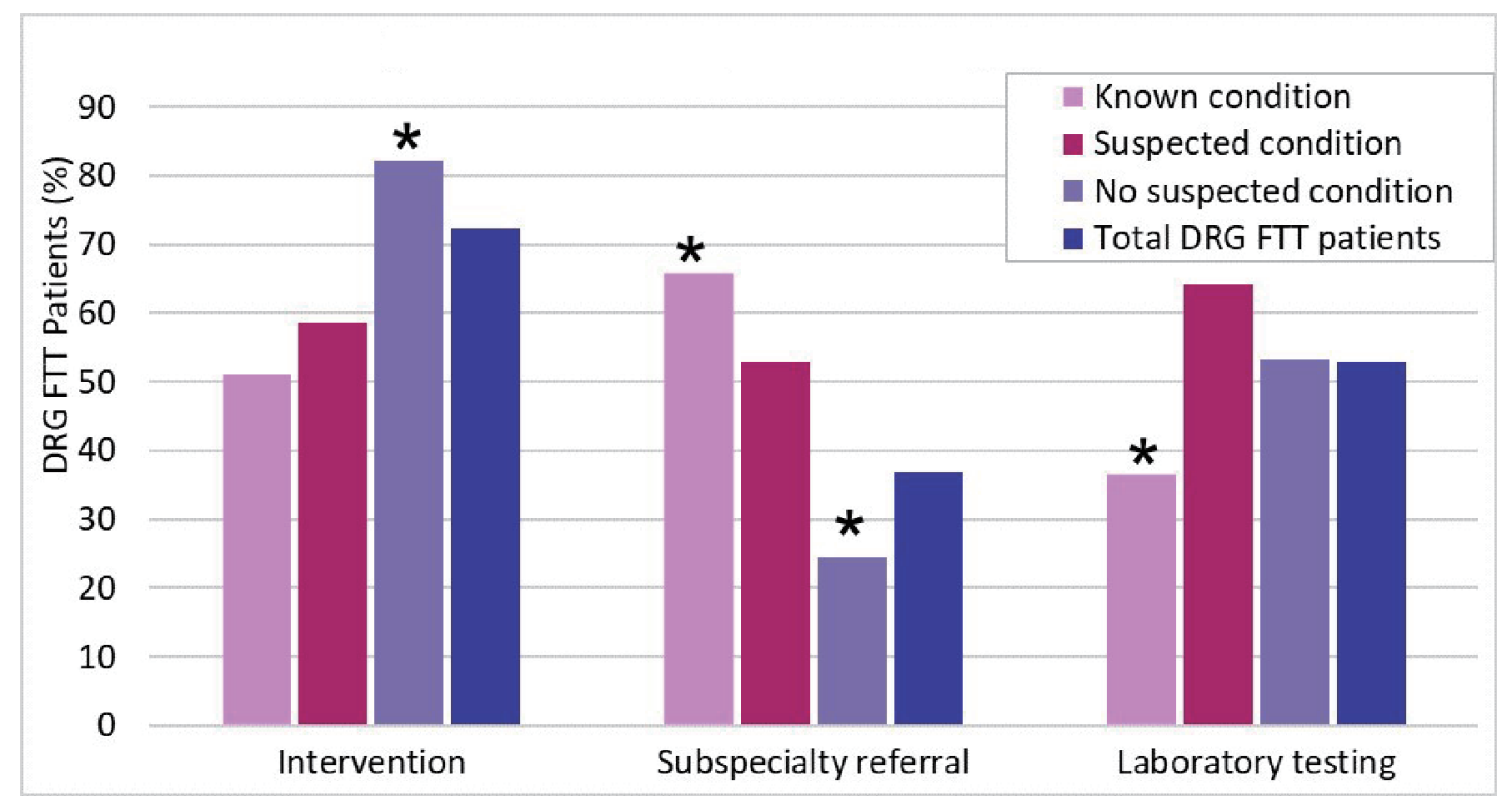

Figure 2. Management at DRG visit. FTT: failure to thrive; DRG: Diagnostic Referral Group. 

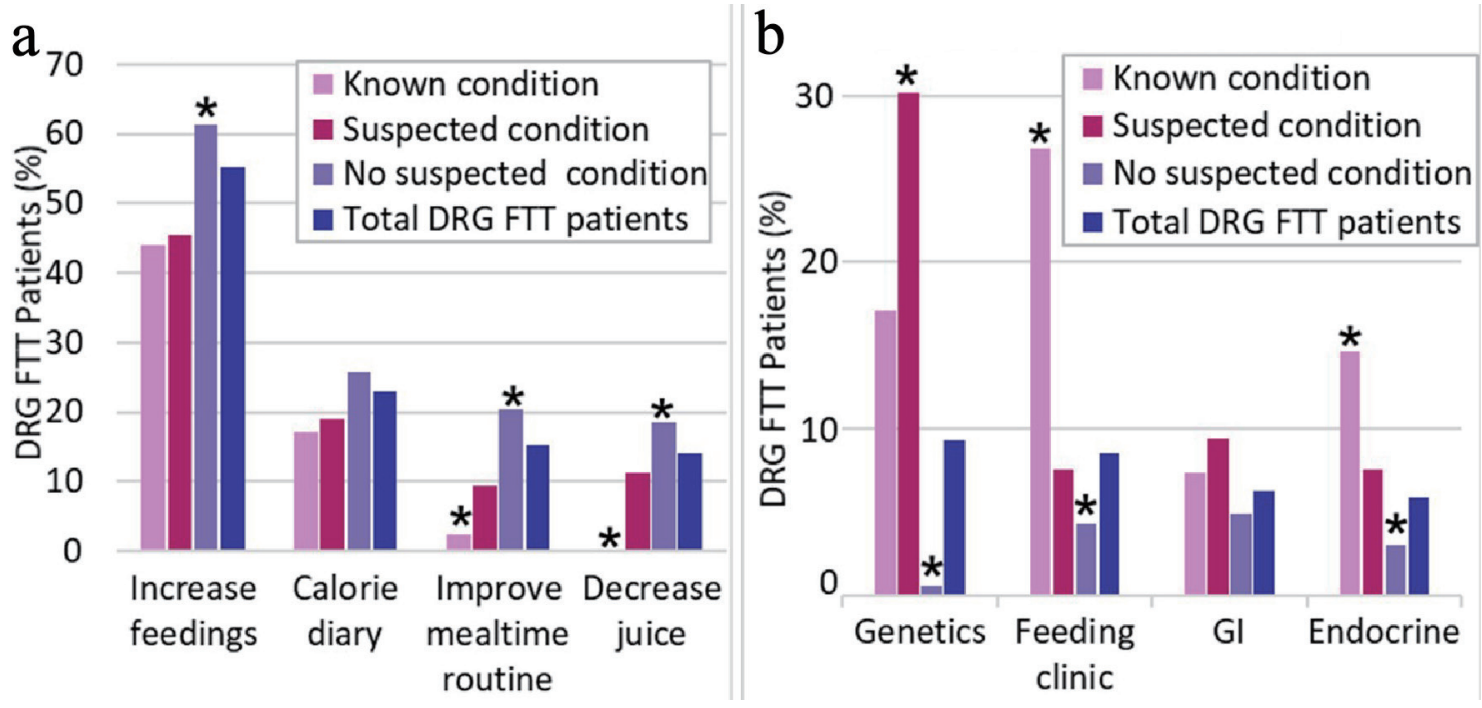

Figure 3. (a) DRG interventions. (b) DRG referrals. Diagnostic Referral Group.

Subspecialty evaluation was recommended least commonly, in only 95 (37.0\%, Fig. 2), more in patients with known conditions $(27,65.9 \%)$. Patients with known conditions were referred to a multidisciplinary feeding clinic $(11,26.8 \%)$ while those with suspected underlying conditions were referred to genetics $(16,30.2 \%)$ and gastroenterology $(5,9.4 \%$, Fig. $3 b)$.

\section{Discussion}

These findings are a step towards filling the informational gap on the escalation of care by assessing the main concerns driving primary care FTT evaluation. The preference for subspecialist evaluation indicates the concern for a single organ system and for an undiagnosed medical condition. The uncertain diagnosis as motivating factor for referral is concerning due to the breadth of literature demonstrating that over $80 \%$ of pediatric FTT is not due to a medical underlying diagnosis [1-3]. This study did not capture if concern is due to clinical features, time pressures in primary care, restricted nutritional history, or medico-legal issues around a missed diagnosis. Regardless of the reason, the concern over underlying diagnosis reflects the hesitancy to diagnose nutritional causes, in favor of making the nutritional cause a diagnosis of exclusion. Feedback from this study may be used to optimize community physician outpatient treatment of FTT by providing tools for identification of high-risk FTT patients and emphasizing critical behavioral interventions.

The diagnostic clinic managed these referred FTT patients and maintained hospitalization rates similar to overall pediatric rates, despite the increased complexity of cases. Our characterization of the referral population replicated previous studies with the assessment that most cases were nutritional and caloric in nature, and would benefit most from nutritional interventions. This replicates the previously demonstrated emphasis on behavioral interventions and nutritional etiologies of FTT even in patients with demonstrated genetic and congenital reasons to be failing to thrive.

The hour-long diagnostic clinic visit enabled thorough assessments to generate tailored medical and behavioral intervention strategies, incorporating both nutritional and behavioral aspects, likely an advantage in comparison to time pressures of primary. The diagnostic clinic recommended interventions with the goal of improving nutritional caloric intake and assessing what the patients were actually consuming via calorie diary. These interventions, though not uniformly applied across the FTT patients, provided effective outpatient treatment for the FTT patients, as shown by the overall improvement in weight-for-age z-scores over diagnostic clinic follow-up and comparable hospitalization rates. This strongly replicates the existing pediatric FTT literature which emphasizes nutritional interventions to achieve weight gain, which has been suggested to preclude extensive medical evaluation.

This study is limited by only studying community provider responses on a subset of their FTT patients. By conducting a survey, we incurred a response bias since only those with an active clinic, the time and motivation to respond to the survey responded. The survey itself depended on a hypothetical question, which was vulnerable to hindsight bias given that community physicians were aware of recommendations from the diagnostic clinic at the time of survey. The inability to link provider responses and specific concerns to patient outcomes limits our ability to interpret whether community physician concerns were substantiated in diagnostic clinic findings. The retrospective chart review was limited to results within out electronic health records and did not capture records from outside health systems.

\section{Conclusions}

This study found that primary providers who referred pediatric FTT patients to a diagnostic clinic would have otherwise favored FTT management utilizing referral to subspecialists, 
driven by concern for an undiagnosed medical condition. This suggests that nutritional causes remain viewed as a diagnosis of exclusion. More investigation is needed to establish if primary providers would be empowered to support nutritional causes if given standardized assessments to evaluate the nutritional and psychosocial contributors to patients' FTT.

\section{Supplementary Material}

Suppl 1. Questionnaire provided to community PCPs.

\section{Acknowledgments}

We thank the support provided from the University of Pittsburgh, School of Medicine, Longitudinal Research Program.

\section{Financial Disclosure}

The study is supported by the Dean's Summer Research Program at University of Pittsburgh School of Medicine.

\section{Conflict of Interest}

None to declare.

\section{Informed Consent}

Informed consent was waived by IRB.

\section{Author Contributions}

GL and TT contributed to the study design. GL planned and implemented the research, analyzed the results, and wrote the manuscript with input from TT.

\section{Data Availability}

Any inquiries regarding supporting data availability of this study should be directed to the corresponding author.

\section{References}

1. Gahagan S. Failure to thrive: a consequence of undernutrition. Pediatr Rev. 2006;27(1):e1-11.

2. Cole SZ, Lanham JS. Failure to thrive: an update. Am Fam Physician. 2011;83(7):829-834.

3. Wright CM. Identification and management of failure to thrive: a community perspective. Arch Dis Child. 2000;82(1):5-9.

4. Lezo A, Baldini L, Asteggiano M. Failure to thrive in the outpatient clinic: a new insight. Nutrients. 2020;12(8):2202.

5. Larson-Nath C, St Clair N, Goday P. Hospitalization for failure to thrive: a prospective descriptive report. Clin Pediatr (Phila). 2018;57(2):212-219.

6. Daniel M, Kleis L, Cemeroglu AP. Etiology of failure to thrive in infants and toddlers referred to a pediatric endocrinology outpatient clinic. Clin Pediatr (Phila). 2008;47(8):762-765.

7. Larson-Nath CM, Goday PS. Failure to Thrive: A Prospective Study in a Pediatric Gastroenterology Clinic. J Pediatr Gastroenterol Nutr. 2016;62(6):907-913.

8. Vellody K, Zitelli BJ. Consultative pediatrics in the new millenium. J Hosp Med. 2010;5(1):E34-40.

9. Gartner JC, Jr., Zitelli BJ, Malatack JJ, Urbach AH, McGregor RS. Consultative pediatrics: a role for the generalist in an academic setting. J Pediatr. 1988;112(6):10351038.

10. Atalay A, McCord M. Characteristics of failure to thrive in a referral population: implications for treatment. Clin Pediatr (Phila). 2012;51(3):219-225. 